\title{
Peliosis hepatoesplénica por enfermedad por arañazo de gato. Reporte de caso
}

\section{Hepatosplenic peliosis by cat scratch disease: report of a case}

\section{Correspondencia}

Eduardo Carcausto Huamaní dr.eduardo.carcausto@gmail.com

Recibido: 10/10/2016 Aprobado: 30/11/2016

Citar como: Carcausto Huamaní E. Peliosis hepatoesplénica por enfermedad por arañazo de gato. Reporte de caso. Acta Med Peru. 2016;33(4):309-12

\section{Eduardo Carcausto Huamaní ${ }^{1}$}

1 Clínica Ricardo Palma. Lima, Perú.

\section{RESUMEN}

Se presenta el caso de una paciente mujer de 73 años que acude por disnea progresiva, fiebre diurna, hiporexia y cefalea de diez días de evolución. Antecedente de neoplasia ovarica, gastritis crónica y tromboembolia pulmonar. La fiebre continuó a pesar de tratamiento antibiótico convencional. Al examen, se identificó compromiso pulmonar y adenopatías inguinales, estas últimas corroboradas por ecografía de partes blandas. Los cultivos realizados fueron negativos y el estudio radiológico mostró compromiso pleuroparenquimal en base de pulmón izquierdo. La tomogragía abdominal evidenció hepatoesplenomegalia con hipodensidades múltiples (peliosis); la serología fue compatible con Bartonella henselae. La paciente evolucionó favorablemente luego de 72 horas de iniciado en tratamiento con doxiciclina a $100 \mathrm{mg}$ cada doce horas.

Palabras clave: Peliosis hepática; Bartonella henselae; Fiebre; Infecciones por Bartonella (fuente: DeCS BIREME).

\section{ABSTRACT}

This is the case of a 73-year-old female patient who presented with progressive dyspnea, diurnal fever, hyporexia and headache lasting for 10 days. She had a past medical history of an ovarian neoplasia, chronic gastritis and pulmonary thromboembolism. Fever continued despite conventional antibiotic treatment. Her physical examination revealed pulmonary involvement and bilateral inguinal adenopathy, the latter was corroborated with soft tissue ultrasound. Cultures performed were reported as negative and a chest X-ray film showed pleuro-parenchymal involvement in the left pulmonary base. An abdominal CT scan showed hepatosplenomegaly with multiple hypodense images (peliosis); and serological studies were compatible with Bartonella henselae. Doxyciclin $100 \mathrm{mg}$ every 12 hours was started, and the patient started recovering uneventfully 72 hours after therapy was instituted.

Keywords: Peliosis hepatis; Bartonella henselae; Fever; Bartonella infections (source: MeSHNLM). 


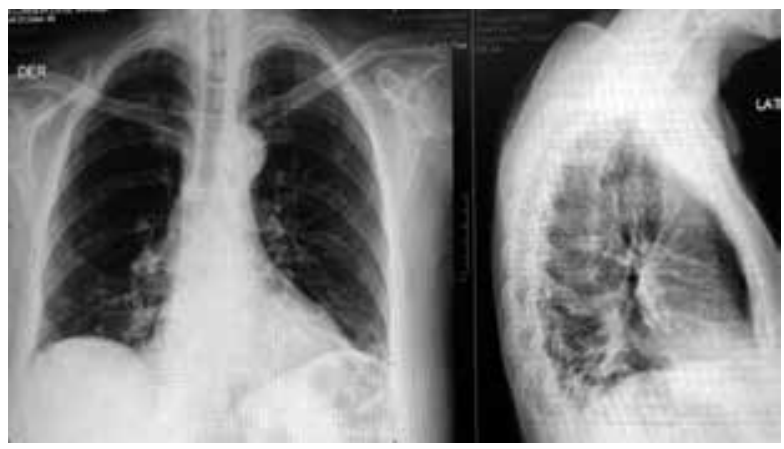

Figura 1. Radiografía de torax (18/5/2015): Leve acentuación de la trama broncovascular en forma difusa bilateral. Atelectasia laminar en base pulmonar izquierda.

\section{INTRODUCCIÓN}

La enfermedad por arañazo de gato (EAG) es ocasionada por la Bordetella henseale y la Bordetella clarridgeiae. Es más frecuente en niños y adultos jóvenes, y clásicamente en $88 \%$ de los casos se describe con la presencia de una pápula de aparición de tres a diez días luego del contacto con el animal, aparición de adenopatía regional entre la semana uno a siete, y fiebre. En el resto de casos hay presentaciones clínicas variadas con compromiso ocular, hepático, óseo; y es una de las causas a considerar en un paciente con fiebre de origen desconocido (FOD) ${ }^{[1]}$.

Desde el año 1996 se reportan en el Perú casos aislados de enfermedades ocasionadas por Bartonella henselae; existen un estudio retrospectivo realizado por Rivera (1999) y otro prospectivo realizado por Huarcaya y Maguiña, quienes reportaron 14 casos confirmados a Bartonella henseale y Bartonella Clarridgeiae ${ }^{[2]}$.

El diagnóstico es difícil por la carencia de métodos moleculares en nuestro medio y la dificultad técnica para aislar la bacteria. Se dispone de serología recientemente con una especificidad de $99 \%{ }^{[3]}$, aunque hasta un $50 \%$ de los casos no desarrollan anticuerpos IgM detectables.

\section{REPORTE DE CASO}

Paciente mujer de 73 años de edad, natural de Ancash, procedente de Lima, con historia de diez dias de disnea progresiva, hiporexia, fiebre diurna cuantificada en domicilio y cefalea. La paciente tenía el antecedente de cistoadenoma ovárico hace tres años que requirió cirugía, con remisión luego sesiones de quimioterapia hasta hace siete meses. Además, tromboembolia pulmonar en dos ocasiones (2009 y 2013) por lo cual estuvo anticoagulada con warfarina hasta cinco meses antes del ingreso. Gastritis crónica y osteoartrosis.

Al examen físico se encontró taquicárdica, febril; piel sin lesiones, tejido celular subcutáneo con turgor disminuido; orofaringe no congestiva, cuello y axilas sin adenopatías, pulmones con

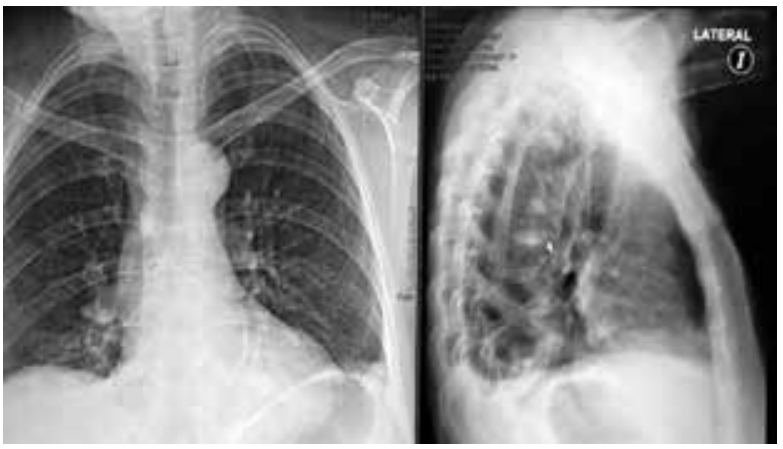

Figura 2. Radiografía de torax (21/5/2015) Opacidad heterogénea con borramientos de ambos senos costodiafragmático a predominio izquierdo a descartar patología pleuroparenquimal.

escasos roncantes; cardiovascular ruidos cardíacos rítmicos, no soplos. El abdomen era blando, distendido, timpánico, doloroso en epigastrio, no rebote. Se palpaban adenopatias inguinales derechas. En el examen neurológico se encontraba lúcida, orientada, sin signos meníngeos ni de focalización.

En sus exámenes de laboratorio se observaron: leucocitos: 8800 $\mathrm{cel} / \mathrm{mm}^{3}$, hemoglobina: $11,4 \mathrm{~g} / \mathrm{dl}$, proteina $C$ reactiva: $25 \mathrm{mg} /$ $\mathrm{dL}$ al ingreso, que se elevó hasta $104 \mathrm{mg} / \mathrm{dL}$ posteriormente. Plaquetas: 128 000/ $\mathrm{mm}^{3}$; aspartato aminotransferasa: 37 $\mathrm{U} / \mathrm{L}$, alanina aminotransferasa: $35 \mathrm{U} / \mathrm{L}$, creatinina: 0,75 mg/dl, albumina: 3,8 mg/dl. Examen de orina sin alteraciones.

Ecografia de partes blandas: en región inguinal derecha se aprecian adenopatías de aspecto inflamatorio siendo la mayor de $33 \times 9 \mathrm{~mm}$.

En la radiografía de tórax de frente y perfil (Figuras 1 y 2 ) se observó compromiso pleuroparenquimal en base de pulmón izquierdo.

Inicialmente se trabajó con el diagnóstico presuntivo de neumonía de la comunidad por lo que recibió tratamiento con ceftriaxona y levofloxacino sin respuesta clínica. Por el antecedente de tromboembolia pulmonar previa se decidió solicitar angiotomografía que evidenció derrame pleural y confirmó el infiltrado parenquimal en la base de pulmón izquierdo.

Se hizo cambio de antibióticos a ceftazidime por vía intravenosa al tercer día ante deterioro clínico y persistencia de fiebre por sospecha de gérmenes resistentes. Se ampliaron los estudios y se consideró al caso como una FOD. Los hemocultivos (dos) tomados tuvieron resultados negativos, así como estudios inmunológicos no significativos clínicamente, anticuerpos antinucleares $(+)$ 1/80 patrón moteado, anticuerpos anticitoplasma de neutrófilos (-), anticuerpos antipéptidos cíclicos citrulinados (-), factor reumatoide: $29 \mathrm{UI} / \mathrm{mL}$ (Valor normal [VN]: $<20 \mathrm{UI} / \mathrm{mL})$.

Se solicitó ecocardiograma en búsqueda de endocarditis; no se encontraron vegetaciones ni masas intracardiacas. 


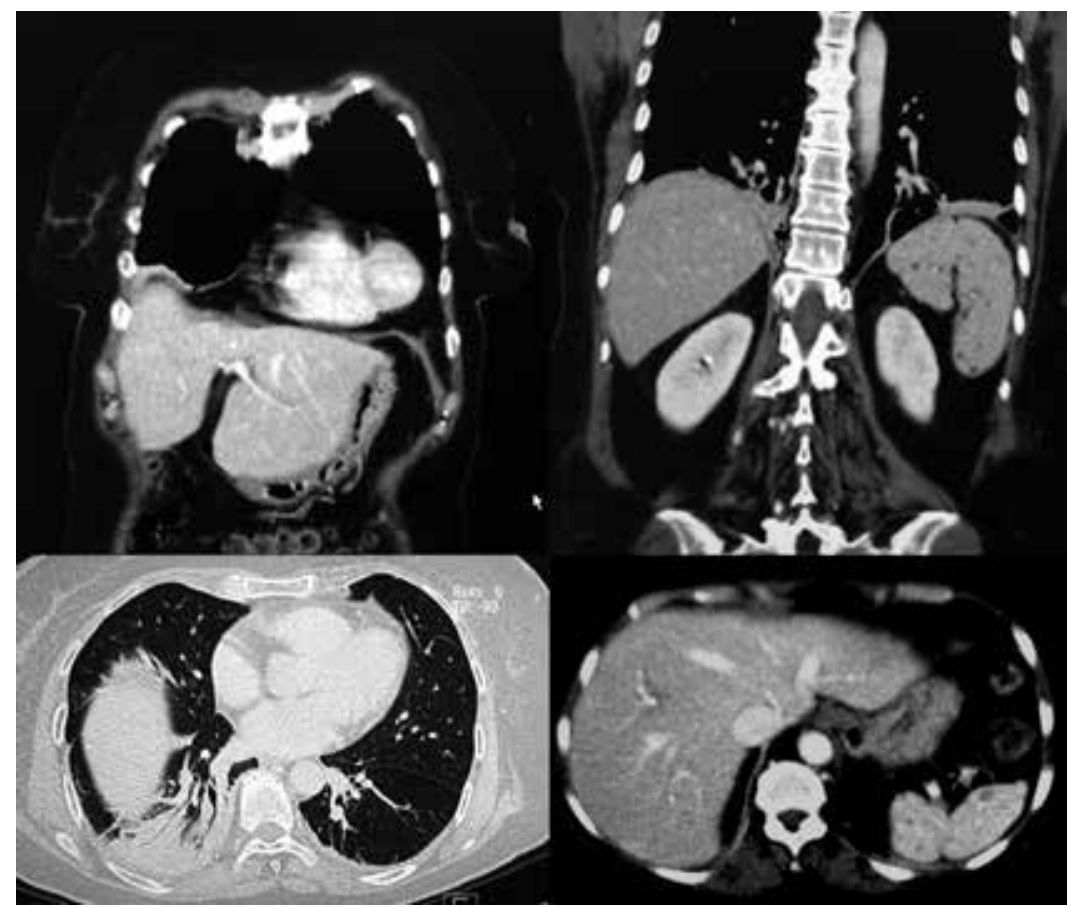

Figura 3. TEM abdomen con contraste (28/5/2015): Consolidación parénquima basal posterior bilateral. Múltiples hipodensidades esplénicas a descartar microabscesos, hepatomegalia a predominio lobúlo izquierdo.

En la tomografía abdominal (Figura 3) se observó en bazo múltiples hipodensidades de aparición reciente -contaba con una tomografía tomada 20 días antes del ingreso en donde no habían lesiones-. Se solicitó evaluación por Infectología. Paciente refería contacto con gatos de la calle un mes antes del ingreso, antes de iniciar los síntomas. Ante la persistencia de fiebre y el antecedente epidemiológico se sospechó infección por $B$. henselae. Se solicitó serologia que mostró valores de lgG/ IgM: 62,0 (VN: 0-15,0), IgM 10,0 (VN: <15).

Se inició tratamiento con doxiciclina $100 \mathrm{mg}$ cada 12 horas, y se descontinuaron otros antibióticos. Luego de 72 horas de tratamientola fiebre cedió y hubo mejora en el estado general. Fue dada de alta con la indicación de que complete el tratamiento por seis semanas.

Dentro del seguimiento, la paciente acudió por emergencia dos semanas después por epigastralgia y náuseas, que ocurrieron debido al uso de doxiciclina; se tomó ecografía abdominal: no hepatoesplenomegalia, no hipodensidades.

\section{DISCUSIÓN}

La peliosis es una enfermedad inusual caracterizada por la presencia de cavidades quísticas irregulares, llenas de sangre. El término proviene del griego pelios que significa oscuro o purpúreo y proviene de la apariencia macroscópica de las lesiones. Se ha identificado asociaciones causales con el uso de anticonceptivos orales o esteroides anabólicos, enfermedades como la tuberculosis o la infección por virus de inmunodeficiencia humana (VIH).
También ha sido asociada con enfermedades hematológicas como la enfermedad de Hodgkin, mieloma y anemia aplásica, cáncer avanzado; el uso de contrastes, y ciertas infecciones virales. En todos estos casos se comprometen el hígado y el bazo ${ }^{[4]}$. También se ha descrito en lesiones tipo proliferación vascular como la angiomatosis bacilar o angiosarcomas ${ }^{[5]}$.

En el caso de la paciente siendo adulta mayor y con antecedente de cáncer, dados los sintomas y el compromiso pulmonar se trató inicialmente como una neumonía de la comunidad; sin embargo al no mejorar clinicamente, elevarse los marcadores inflamatorios y no teniendo otro foco evidente, se cambió la antibioticoterapia a una cefalosporina con actividad para gérmenes nosocomiales. Después de 48 horas de iniciarla y como parte del estudio de FOD se encontraron imágenes compatibles con peliosis esplénica, y agregado al hallazgo de adenopatia inguinal, se sospechó también en una infección por alguna especie de Bartonella. Ampliando la anamnesis se encontró la exposición a gatos. Las infecciones producidas por Bartonella son transmitida por varios vectores que provocan diversos cuadros clínicos. La infecciones más frecuentes, aparte de la EAG, son la enfermedad de Carrión (causada por la B. bacilliformis), fiebre de las trincheras (causada por la B. Quintana), endocarditis y neuroretinitis (causada por la B. elizabethae), angiomatosis bacilar y peliosis hepática en hospederos inmunocomprometidos ocasionados por $B$. henselae (53\%) y B quintana, (43\%) según series de casos ${ }^{[6]}$.

En general, la EAG tiene una incidencia incierta aún en países desarrollados, que afecta principalmente la población pediátrica ${ }^{[1]}$. El caso presentado corresponde a una mujer adulta mayor con 
un nivel de inmunodeficiencia posterior a una remisión reciente de una neoplasia ovárica lo cual podría explicar la presentación inusual de compromiso hepatoesplénico en el contexto de una EAG atipica, además alteraciones inmunológicas que podrían suscitarse por ser adulta mayor y que la harían susceptible a un cuadro de mayor severidad.

La incidencia de peliosis bacilar, forma visceral de angiomatosis bacilar, también es desconocida; predomina en inmunosuprimidos -especialmente en pacientes con $\mathrm{VIH}$-. En series de casos de EAG, la hepatomegalia ocurrió en el $18 \%$ de los $\operatorname{casos}^{[7]}$ mientras que entre $5-14 \%$ presentan compromiso sistemico y severo ${ }^{[8]}$.

El aislamiento de la $B$. henselae es dificultoso y no disponible en nuestro centro.Se optó por enviar nuestra muestra para la detección de anticuerpos que por serología aunque la sensibilidad y especificidad altas se asocian a valores mayores a $1 / 64$ para $\lg G$, considerando que hasta $10-15 \%$ de casos no se detectan anticuerpos y este porcentaje aumenta en inmunosuprimidos y adultos mayores.

En nuestro caso, la sospecha de infección por $B$. henselae se confirmó por el antecedente epidemiologico, la presencia de adenopatías regionales, serología positiva y respuesta clínica a doxiciclina.

Este es un caso inusual en una persona adulta mayor con infección sistémica. En cuanto al tratamiento se indicó tetraciclinas con buen resultado. Otras opciones de tratamiento en casos de angiomatosis y peliosis bacilar incluyen la combinación de rifampicina mas doxiciclina ${ }^{[8]}$.

Debido a la presencia de gastritis crónica la paciente regresó dos semanas después con molestias gastricas por la doxiciclina que fueron resueltas, completando el tratamiento y curación de cuadro infeccioso.
Agradecimientos: Al Dr. Carlos Seas, por su invaluable apoyo y ayuda en el caso.

Fuente de financiamiento: El presente estudio ha sido autofinanciado por los autores.

Declaración de conflictos de intereses: Los autores declaran no tener conflictos de interés con respecto a la publicación del presente estudio.

\section{REFERENCIAS BIBLIOGRÁFICAS}

1. Polanco Aguilar PM, Cornejo Giraldo M, Zapata Aguilar $\mathrm{E}_{\text {, Calderón }}$ Arenas VH, Márquez Díaz P, Maguiña Vargas C. Primer reporte de enfermedad sistémica por arañazo de gato (Bartonella henselae) en el Perú. Acta Med Peru. 2008;25(4):228-31.

2. Huarcaya E, Maguiña C, Merello J, Cok J, Birtles R, Infante B, et al. A prospective study of cat-scratch disease in Lima-Peru. Rev Inst Med Trop S Paulo. 2002;44(6):325-30.

3. Harrison TG, Doshi N. Serological evidence of Bartonella spp. infection in the UK. Epidemiol Infect. 1999;123(2):233-40.

4. Davidson J, Tung K. Splenic peliosis: an unusual entity. Br J Radiol. 2010;83(990):e126-8.

5. Chieng GH, Zanetto $U$, Harper E. Splenic peliosis: an incidental finding. BMJ Case Rep. 2010;2010:bcro420102935. doi:10.1136/ bcr.04.2010.2935

6. Vásquez T P, Chanqueo C L, García C P, Poggi M H, Ferrés G M, Bustos $\mathrm{M} M$, et al. Angiomatosis bacilar por Bartonella quintana en un paciente con infección por virus de inmunodeficiencia humana. Rev Chilena Infectol. 2007;24(2):155-9.

7. Maguiña C. Las nuevas y viejas bartonellas:una enfermedad emergente mundial. Acta Med Peruana. 2001;18(3):122-32.

8. Rolain JM, Brouqui P, Koehler JE, Maguiña C, Dolan MJ, Raoult $D$. Recommedations for treatment of human infections caused by Bartonella species. Animicrob Agents Chemother. 2004; $48(6) 1921-33$

\section{Las ediciones anteriores de Acta Médica Peruana están disponibles en:}

\section{www.redalyc.org}

